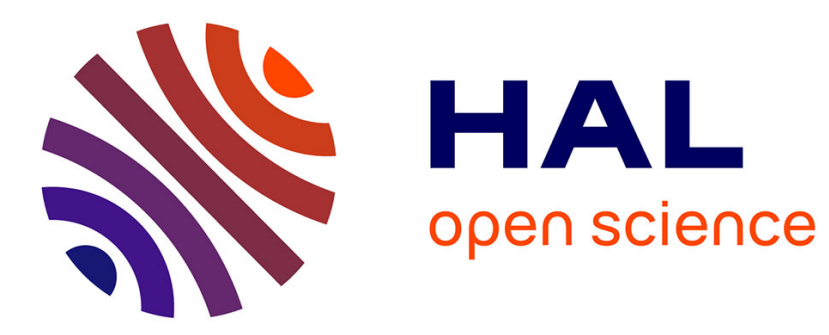

\title{
Concentration d'entreprises et innovation : Esquisse d'une typologie des systèmes productifs locaux Michel Grossetti
}

\section{To cite this version:}

Michel Grossetti. Concentration d'entreprises et innovation: Esquisse d'une typologie des systèmes productifs locaux. Géographie, Économie, Société, 2004, Vol. 6 (n² 2), p.163-177. halshs-00476944

\section{HAL Id: halshs-00476944 \\ https://shs.hal.science/halshs-00476944}

Submitted on 27 Apr 2010

HAL is a multi-disciplinary open access archive for the deposit and dissemination of scientific research documents, whether they are published or not. The documents may come from teaching and research institutions in France or abroad, or from public or private research centers.
L'archive ouverte pluridisciplinaire HAL, est destinée au dépôt et à la diffusion de documents scientifiques de niveau recherche, publiés ou non, émanant des établissements d'enseignement et de recherche français ou étrangers, des laboratoires publics ou privés. 


\title{
Concentration d'entreprises et innovation : \\ Esquisse d'une typologie des systèmes productifs locaux
}

\author{
Michel Grossetti \\ Centre d'étude des rationalités et des savoirs (Cers) \\ Université de Toulouse-le-Mirail \\ 5, Allées A. Machado \\ 31058 Toulouse Cedex \\ Tél : 0561503669 \\ Fax : 0561503870 \\ E-mail : Michel.Grossetti@univ-tlse2.fr \\ Géographie, économie, société, Vol. 6, n².
}

\section{Résumé}

Cet article a pour objectif d'élargir la notion de «système productif local » au-delà du cas particulier des «districts» de petites et moyennes entreprises spécialisées dans des produits traditionnels. Il s'appuie pour cela sur le critère de l'existence entre les entreprises d'un espace déterminé (et éventuellement des organisations scientifiques), de coopérations coexistant avec des relations de compétition. Ces coopérations sont favorisées principalement par la structure des réseaux sociaux. Sur la base de cette conception générale, il est possible d'utiliser la notion de système productif local pour analyser diverses formes productives (technopoles, systèmes locaux de sous-traitance, activités rares à marché mondial, etc.).

Mots clés : systèmes productifs locaux ; réseaux sociaux ; technopoles ; districts ; proximité

\section{Outline of a typology of local productive systems}

\begin{abstract}
The aim of this article is to broaden the notion of «local productive system » beyond the specific case of «districts » of small firms specialized in traditional products. I use the criterion of the existence of cooperations that coexist with competition relations within a set of firms of a specific place (and sometimes scientific organizations). These cooperations are mainly favoured by the structure of social networks. On the basis of this general conception, it is possible to use the notion of local productive system for analysing different productive forms (technopoles, local subcontracting systems, rare activities with mondial market, etc.).
\end{abstract}

Keywords : local productive systems ; social networks ; technopoles ; districts; proximity 
L'idée selon laquelle la concentration spatiale peut favoriser des échanges entre entreprises débouchant sur la formation de systèmes locaux a émergé depuis une vingtaine d'années à partir de travaux empiriques qui font apparaître sous diverses désignations (districts, milieux innovateurs, systèmes productifs locaux, etc.) des concentrations d'entreprises, et parfois aussi d'institutions publiques d'enseignement supérieur ou de recherche, entretenant entre elles des relations permettant entre autres la mise en commun de savoirs techniques et donc la création ou la diffusion d'innovations.

Les situations décrites sont très diverses, allant des systèmes composés de soustraitants, rassemblés autour d'un grand donneur d'ordre dans des sites industriels traditionnels, à des industries culturelles concentrées dans certains grands centres urbains. Toutefois, deux catégories de cas ont été particulièrement étudiées. La première est constituée des réseaux locaux de petites entreprises d'industrie traditionnelle (textile, chaussures, ameublement, etc.), baptisés par certains auteurs «districts industriels » en référence aux travaux d'Alfred Marshall, l'exemple le plus cité étant celui des sites de la «Troisième Italie» (Beccatini, 1989, 1992 ; Capecchi, 1987). D'autres auteurs ont défini ces concentrations de petites entreprises comme des «systèmes industriels locaux» (Raveyre et Saglio, 1984) ou comme des «systèmes productifs locaux »(Courlet et Pecqueur, 1991). Les systèmes relevant de la seconde catégorie rassemblent des entreprises dont l'activité comporte une part élevée de recherche et développement, le plus souvent en relation avec des organisations scientifiques, le cas le plus célèbre étant celui de la Silicon Valley (Rogers et Larsen, 1984 ; Saxenian, 1981, 1994). Ils ont selon les cas été qualifiés de «districts de haute technologie » (Saxenian, 1994), de «technopoles » (Boisgontier et de Bernardy, 1988 ; Benko, 1991), de «systèmes locaux d'innovation » (Gilly et Grossetti, 1993).

Ces formes économiques spatiales ont été étudiées ou commentées par de si nombreux auteurs que l'on pourrait avoir l'impression que tout a été dit sur ce thème. Cependant, ces auteurs sont issus de disciplines différentes (économie régionale ou industrielle, géographie économique, sociologie industrielle), raisonnent à partir d'horizons théoriques souvent peu compatibles et travaillent avec des méthodes diverses, ce qui rend parfois confuse la perception d'ensemble du phénomène. 
Mon objectif ici est de proposer un cadre synthétique d'appréhension de ces formes locales de coopération en repartant des études empiriques. Celles-ci s'accordent sur l'existence d'échanges au moins partiellement non marchands entre les organisations de ces systèmes, échanges ayant entre autres pour effet le partage, la diffusion ou la construction commune de savoirs techniques ou économiques. Cela favorise en général l'apparition d'innovations ou, au moins, leur diffusion au sein des systèmes locaux. Quelle que soit la façon dont ces concentrations d'activités se sont réalisées, il semble donc que tout se passe comme si, «toutes choses non spatiales que l'on ait pensé à mesurer égales par ailleurs », la proximité spatiale favorisait, dans certaines conditions, des échanges entre les organisations, propices à l'apparition ou la diffusion d'innovations. Lorsque cela se produit, les études empiriques les plus convaincantes montrent que les échanges entre organisations sont sous-tendus par des réseaux sociaux interindividuels.

Si l'on fait de la coopération locale entre entités indépendantes et éventuellement concurrentes le principe commun de ces deux catégories de systèmes, il est certainement possible de l'élargir à d'autres types de situations. Parmi la large gamme des désignations utilisées, j'ai choisi celle de «système productif local » comme terme générique pour désigner de tels systèmes parce qu'elle me paraît la plus juste, même si l'existence de politiques publiques assimilant les systèmes productifs locaux au cas des districts rend nécessaire une explicitation.

Dans cet article, je voudrais d'une part préciser ce qui peut être commun à toutes les configurations comportant des coopérations locales, et d'autre part élaborer une typologie des configurations selon les contextes et les types d'entreprises impliquées. Il est d'abord nécessaire de revenir sur les travaux traitant des districts industriels ou des technopoles pour clarifier la base du raisonnement. Je développerai ensuite l'explication des effets de proximité par l'encastrement des logiques organisationnelles dans les réseaux sociaux, explication que je considère comme la mieux fondée empiriquement. Ensuite, je tenterai d'élargir la notion et d'ébaucher une typologie.

\section{Des districts aux systèmes productifs locaux}


Les travaux empiriques sur les concentrations industrielles formant système sont à la fois très nombreux et très variés. Construits autour de problématiques diverses, ils ne sont pas toujours très précis sur la nature et le contenu des relations observées. C'est pourquoi je me concentrerai sur ceux qui sont les plus explicites sur ce plan pour tenter de définir le problème de la façon la plus précise possible, en commençant par les districts pour aborder ensuite les systèmes locaux de recherche et développement et les études sur les relations science - industrie.

\subsection{Les districts}

Une des études les plus abouties sur les systèmes de type «districts » est présentée dans l'article de Marie-Françoise Raveyre et Jean Saglio (1984) sur les petites et moyennes entreprises de plasturgie d'Oyonnax ${ }^{1}$. Tout en étant concurrentes sur les prix, ces entreprises coopèrent pour la diffusion des techniques, voire la gestion de certains marchés. Raveyre et Saglio expliquent ce phénomène par l'existence de normes sociales («il existe des règles communément admises, qui dictent les comportements et modèlent les relations », écrivent-ils par exemple à propos des relations de soustraitance, p.166). La notion de système industriel local avancée par les auteurs intègre les relations entre entreprises (concurrence et coopération), les relations professionnelles, les rapports aux pouvoirs publics et la gestion de l'innovation et des techniques. Des situations tout à fait comparables ont été observées à peu près à la même époque dans certaines parties de l'Émilie-Romagne en Italie (Beccatini, 1989 ; Capecchi, 1987) ou dans l'Arc jurassien en Suisse (Maillat, Crevoisier et Vasserot, 1992). Puisant dans la tradition de leur discipline, certains des économistes italiens travaillant sur des cas similaires ont eu recours à la notion un peu oubliée de « district industriel », utilisée naguère par A. Marshall (1890). Dans cette conception, la concentration spatiale constitue une forme de substitut à la concentration des entreprises, les interactions locales permettant de produire des économies d'échelle équivalentes à celles que permet la grande entreprise. Certains observateurs des districts

\footnotetext{
${ }^{1}$ La recherche dont rend compte cet article fait partie d'un ensemble de travaux conduits par les sociologues lyonnais, dans le cadre d'un programme de recherche du CNRS consacré à la croissance urbaine. Une autre de ces recherches, consacrée à Annonay, a servi de base à l'ouvrage de Bernard Ganne, Gens du cuir, gens du papier, Éd. du CNRS, 1983.
} 
italiens insistent, comme les sociologues lyonnais, sur les normes sociales locales (voir Beccatini,1992, par exemple).

Dans ces travaux, les normes ou règles locales sont implicitement associées aux réseaux sociaux. Ainsi, dans l'article de Marie-Françoise Raveyre et Jean Saglio, chaque fois que l'affirmation de l'existence de ces règles est assortie d'un exemple, celui-ci renvoie à l'existence de réseaux interindividuels (Grossetti, 2001a). Les relations entre entreprises, en particulier ici le partage de savoirs techniques, s'appuient donc sur des relations entre les individus composant ces entreprises, ce qui peut se lire comme un cas classique d' «encastrement » (Granovetter, 1985) mais n'implique pas en soi nécessairement l'existence de règles ou de normes locales spécifiques.

Si nous résumons les traits caractéristiques des districts selon ces travaux, nous avons des petites entreprises d'industrie relativement traditionnelle, qui sont en compétition sur les prix tout en coopérant sur le plan technique. Cette coopération passe par des relations non marchandes et le plus souvent informelles, c'est-à-dire par des relations individuelles.

Voyons à présent le cas des systèmes de firmes dont l'activité implique une forte part de recherche et développement, ce que certains ont parfois baptisé des "districts de haute technologie".

1.2. Les systèmes locaux d'activités de haute technologie et les relations science industrie

Cette seconde catégorie de travaux est amorcée par les études de chercheurs américains sur la Silicon Valley ou d'autres sites de ce pays (Saxenian, 1981 ; Rogers et Larsen, 1984 ; Scott, 1985). Les réseaux de firmes de la vallée, spécialisées dans les semi-conducteurs ou l'informatique, sont constitués de relations clients-fournisseurs qui dépassent le simple accord marchand, les fournisseurs intervenant à diverses étapes du processus de conception : «Les managers décrivent les relations qu'ils ont avec leurs fournisseurs comme impliquant un accord personnel et moral qui va bien au delà des attentes d'une simple relation d'affaire »(Saxenian, 1990b, p.12). Pour expliquer ces 
collaborations, ces auteurs mettent en avant l'importance des réseaux sociaux. Ainsi, pour AnnaLee Saxenian, «la résistance de Silicon Valley doit autant à ses réseaux de relations sociales, professionnelles et commerciales qu'aux efforts des entrepreneurs individuels »(Saxenian, 1990a, p.105).

Les études de chercheurs américains, sur lesquelles l'attention a été attirée par des universitaires francophones installés aux États-Unis (Castells, 1984), et les tentatives d'aménagement de sites comparables en France, dont certaines commencent à être étudiées empiriquement à la fin des années 1970 (Bernardy et Boisgontier, 1976, 1988) finissent par amener les économistes français à s'intéresser à ce type de phénomène. L'économie régionale et l'économie industrielle se mobilisent au début des années 1980 (Bellet et Boureille, 1985 ; Aydalot, 1986 ; Perrin, 1986) pour produire à la fois des études empiriques et des constructions théoriques interprétatives qui tentent de définir des « milieux innovateurs », « réseaux innovateurs » ou autres «technopoles ». Dans un premier article de synthèse sur le cas de la Silicon Valley, Bernard Planque reprend une thèse énoncée par Rogers et Larsen (1984), qui fait de la mise en réseau le principal effet de la concentration au sein d'un «technopôle»: «La proximité physique entre acteurs locaux joue un rôle déterminant dans la constitution de ce réseau d'échanges d'informations qui constitue au sens propre la principale économie externe d'agglomération créée par le technopole en même temps qu'il génère le phénomène de technopolarisation lui-même » (Planque, 1985, p. 922).

\subsection{Les effets de proximité}

Sans passer en revue la totalité de l'abondante littérature économique parue sur ces thèmes depuis les travaux cités, on peut dire que des études empiriques portant sur des systèmes de petites entreprises «traditionnelles » ou sur des concentrations d'activités à forte teneur en recherche et développement convergent sur l'existence de coopérations locales entre firmes (ou entre firmes et organisations scientifiques) ${ }^{2}$. Ce constat empirique est renforcé par les études relatives aux relations entre organisations

$2 \mathrm{Ce}$ constat n'implique nullement qu'il s'agisse là d'une forme de coordination économique plus performante que d'autres. Ces relations locales existent, et notre problème ici est d'en expliquer les logiques de construction. 
scientifiques et industrie (Grossetti, 1995; Estades, Joly et Mangematin, 1996 ; Feldman, 1994 ; Mansfield et Lee, 1996), ou aux collaborations entre chercheurs (Katz, 1994), qui toutes montrent l'importance relative des relations locales ${ }^{3}$.

La proximité spatiale a donc des effets sur les coordinations entre organisations, la probabilité d'existence de relations augmentant, «toutes choses non spatiales égales par ailleurs », lorsque la distance se réduit, le principal effet de seuil observé empiriquement se situant à l'échelle de l'agglomération urbaine ou du bassin d'emplois.

Ce dernier point est important : aucune étude empirique n'a mis en évidence d'effets de proximité spécifiques situés à l'échelle des parcs d'activités technologiques ou de quartiers particuliers. Lorsqu'elle se produit, la concentration de certaines activités à des niveaux infra-urbains est le fruit de logiques d'implantation, que celles-ci soient « spontanées » (en fait guidées généralement par le jeu des réseaux sociaux) ou qu'elles résultent de politiques publiques, comme celles qui consistent à aménager des parcs technologiques ou des zones d'activités. Mais il faut bien distinguer les logiques d'implantation qui aboutissent à concentrer des activités dans certains espaces, avec les effets de proximité qui expliquent la coordination de ces activités. On peut montrer par exemple, dans le cas de villes à forte activité de recherche et développement comme Grenoble et Toulouse, que des logiques historiques d'implantation très différentes aboutissent à une situation similaire sur le plan des effets de proximité (Grossetti, 2001b). Cela ne signifie pas que les logiques d'implantation et les effets de proximité n'ont aucun lien. Les effets de proximité peuvent intervenir dans les logiques d'implantation (une firme peut choisir de s'implanter quelque part pour bénéficier de ces effets), même si les études empiriques sur les choix de localisation montrent que ce phénomène est finalement très limité (voir Carrincazeaux, 1999, pour les activités de recherche et développement par exemple).

Le fait que la ville soit une échelle suffisante pour permettre l'apparition d'effets de proximité permet de comprendre que peuvent parfaitement coexister dans un même espace des systèmes productifs distincts peu liés entre eux. C'est d'ailleurs ce qui se produit dans la plupart des agglomérations urbaines. Les logiques historiques

\footnotetext{
${ }^{3}$ Bien que les espaces pris en compte soient différents (agglomération urbaine dans le cas de la France, État pour les États-Unis, distance kilométrique dans l'étude de Katz).
} 
d'implantation des organisations et des ménages, ainsi que celles des marchés fonciers, peuvent produire la concentration dans un même quartier d'activités formant système, voire la formation d'espaces infra-urbains économiquement homogènes, mais cela n'est en rien nécessaire à l'existence des effets de proximité. Ainsi, dans une ville comme Toulouse, l'installation des activités aéronautiques au nord-ouest, de l'électronique au sud-ouest et de l'industrie spatiale au sud-est est le fruit des politiques d'aménagement urbain des années soixante. Mais cela n'a nullement empêché la formation, sous l'effet conjugué de l'évolution des technologies et des relations science-industrie, d'un système unique dédié aux compétences de conception en électronique et informatique, système qui se déploie sur l'ensemble de l'agglomération, et qui coexiste avec un autre système totalement distinct tourné vers les sciences du vivant et avec d'autres activités, tout aussi distinctes, liées aux fonctions de capitale régionale (Zuliani et Grossetti, 2004). Chacun de ces systèmes se structure autour de réseaux distincts, tant au niveau des organisations qu'à celui des individus.

Au-delà de ces résultats, les études empiriques existantes ne sont pas suffisamment comparables pour qu'il soit possible de quantifier ou modéliser précisément les effets de proximité d'un point de vue général. Il est aussi difficile d'évaluer leur éventuelle variabilité selon les configurations locales. Toutefois, la certitude de leur existence justifie la construction de théories explicatives qui n'ont pas manqué de se multiplier dans les domaines de l'économie industrielle ou régionale.

\section{L'encastrement dans les réseaux sociaux}

Parmi les diverses théories qui ont été avancées pour expliquer les effets de proximité, la mieux fondée empiriquement est celle qui fait résulter la mise en relation des organisations de l'existence de réseaux sociaux locaux interindividuels traversant leurs frontières (Saxenian, 1994 ; Grossetti et Bès, 2002)4.

\footnotetext{
${ }^{4}$ Les études empiriques convergent pour montrer que les réseaux sociaux sont, et de loin, la principale source des effets de proximité, mais il est aussi possible (bien que difficile) de favoriser ceux-ci par des impulsions institutionnelles (spécialistes locaux du transfert de technologie jouant les intermédiaires entre les laboratoires et les entreprises par exemple), voire par certaines médiations plus classiquement marchandes (éditions d'annuaires locaux par exemple). Quantitativement presque négligeables actuellement, ces autres sources de construction de coopérations locales ne le resteront pas
} 
Si les réseaux sociaux expliquent les effets de proximité, c'est qu'ils ont une part locale toujours importante. C'est ce qui ressort des enquêtes sur les réseaux personnels en milieu urbain conduites par Claude S. Fisher (1982) à San Francisco et par Barry Wellman (1979) à Toronto 5 . Pour Fisher, 65\% des relations sont situées dans une aire accessible en une heure de transport à partir du lieu de résidence de l'enquêté. Wellman, lui, compte $75 \%$ des relations dans l'agglomération de Toronto. Il ne s'agit pas en général de relations de voisinage proche, puisque seulement $25 \%$ des personnes citées dans l'enquête de Fisher habitent dans un lieu accessible en moins de cinq minutes (contre $40 \%$ entre cinq minutes et une heure) et $13 \%$ de celles de l'enquête de Wellman sont situées dans le même quartier que la personne interrogée (contre $62 \%$ dans le reste de l'agglomération). Par ailleurs, le passage du quartier à la ville affecte peu la fréquence des contacts, alors que celle-ci chute rapidement pour des distances plus grandes 6 . Une étude sur les réseaux personnels de 300 habitants de l'agglomération de Toulouse en 2001 (utilisant une méthode proche de celle de Fischer) aboutit à des résultats comparables (avec une part locale plus importante) : $83 \%$ des personnes citées habitent à moins d'une heure de transport et $71 \%$ dans les communes de l'agglomération (Grossetti, 2003).

Les réseaux personnels tendent donc à être concentrés en partie dans un espace correspondant aux actuelles agglomérations urbaines. Au sein de cet espace, le voisinage proche représenté par le quartier joue un rôle mineur. Autrement dit, s'ils s'appuient sur les réseaux sociaux, les effets de proximité se situent pour l'essentiel à l'échelle de la ville. Cela correspond bien aux résultats empiriques exposés plus haut.

nécessairement toujours. Toutefois, dans la mesure où elles passent par la formalisation de l'information économique ou stratégique, il sera toujours difficile de les cantonner aux espaces locaux.

5 Wellman a beaucoup insisté sur le caractère «non local» des réseaux sociaux, mais sa définition du local, restreint à l'échelle du quartier, est à replacer dans le cadre spécifique des travaux nord-américains sur les communautés. Pour lui, les relations entre personnes résidant dans la même ville mais dans des quartiers différents sont «non locales». Dans l'optique que je défends ici, des relations externes au quartier dans la même ville sont considérées comme « locales » au sens où l'on observe effectivement une concentration des entourages relationnels à l'échelle des agglomérations.

6 Dans une étude consacrée aux contacts, Wellman indique que le nombre moyen de contacts en face à face est de 143 pour les relations situées dans un rayon de un mile, 123 pour celles qui sont situées entre un et cinq miles et 41 pour les autres. Pour les contacts par téléphone, les moyennes sont de 66, 81 et 34 (Wellman, 1996). 
L'«atmosphère industrielle » de Marshall n'est qu'une métaphore. Pour bénéficier des effets de proximité au sein d'un système productif local, il ne suffit pas d'en respirer l'air plus ou moins pollué, il faut rencontrer des gens, nouer des relations, construire des réseaux. Cela prend du temps et ne s'effectue pas par simple présence dans les lieux.

En effet, la proximité ne crée pas directement des relations. Le simple fait d'habiter dans une ville ou un quartier ne génère pas de liens avec tous les autres habitants, ni même ceux qui fréquentent les mêmes lieux. Les relations interindividuelles naissent le plus souvent au sein de groupes constitués avant de s'autonomiser. Ces groupes constitués peuvent être les familles, les organisations ou plus généralement ce qu'Alain Degenne (Degenne et Forsé, 1994) appelle, d'après Georg Simmel 7 , des cercles sociaux, c'est-à-dire des collectifs dont les acteurs se disent membres, et au sein desquels ils ont des interactions avec d'autres membres. Les méthodes utilisées dans les enquêtes de Fischer et de Wellman ne permettent guère d'aller plus loin qu'une évaluation assez approximative des circonstances de création des relations. Si l'on se fie à celle de Fischer, la plus détaillée, on aurait un tiers de liens familiaux et au moins un autre tiers de relations créées dans le cadre d'organisations diverses allant de l'entreprise à l'association en passant par l'école, le reste se partageant entre les relations suscitées par des intermédiaires, les liens de voisinage et les rencontres d'autres types (hasard, participation à des groupes non organisés, etc.). Dans l'enquête sur les réseaux des Toulousains, $60 \%$ des relations sont issues de contextes collectifs (36\% pour la famille ou l'enfance, $24 \%$ pour les études supérieures, le travail ou les associations), $26 \%$ de la sociabilité (personnes rencontrées par un ami, le conjoint, etc.), et $8 \%$ du voisinage. Seulement $6 \%$ des relations sont nées dans des contextes d'une autre nature, dont les rencontres de hasard ne forment d'ailleurs qu'une petite part.

L'importance prise par les contextes collectifs montre que lorsque l'on s'intéresse à la genèse des relations, la notion d'encastrement fonctionne en quelque sorte à l'envers : ce sont les liens individuels qui sont au départ "encastrés" dans des structures collectives (famille, organisations), dont ils se détachent éventuellement par la suite (ce qui relève d'un processus de "découplage" au sens défini par White, 1992). Les

\footnotetext{
7 Georg Simmel, contemporain d'Émile Durkheim et de Max Weber, est considéré comme l'un des fondateurs de l'approche interactionniste en sociologie.
} 
résultats d'AnnaLee Saxenian sur la Silicon Valley permettent d'appréhender cet aspect de la genèse des relations individuelles. Elle montre en effet que la création et le maintien des relations n'est pas seulement le fait des interactions liées au fonctionnement des entreprises, mais résulte aussi de l'existence d'organisations professionnelles ou commerciales locales, qu'elle a étudiées en tant que telles. Des associations telles que The Semiconductor Equipment and Materials International ou The Software Entrepreneur's Forum organisent des rencontres, des dîners, des séminaires, des stages de formation qui jouent un rôle important dans le développement des réseaux sociaux de la Silicon Valley (Saxenian, 1992).

Le processus de création et de maintien des relations est largement dépendant des activités routinières des individus, organisées ou non. Or, ces activités routinières ne peuvent être trop dispersées. Le critère d'une heure de transport à coût modéré, souvent utilisé pour la taille des agglomérations urbaines, semble bien adapté à cette contrainte spatiale. Nous retrouvons donc les coûts de transport chers aux théoriciens de la localisation des activités, mais cette contrainte s'exerce moins sur les entreprises que sur les individus et leurs relations. De surcroît, cette contrainte n'est qu'un cadre au sein duquel se structurent les systèmes d'activités et les relations qui les accompagnent. Or, ces activités et ces relations dépendent des trajectoires spatiales des individus en question.

La genèse des relations sociales explique que les réseaux individuels aient toujours une part locale importante, mais cette part et la nature des liens locaux dans un site déterminé varient selon les mobilités des acteurs et en particulier selon la durée de leur résidence dans le site (Grossetti, 2003). Le fait d'avoir vécu successivement dans des villes différentes génère des parties de réseau situées dans chacune de ces villes qui sont autant de traces de la trajectoire d'un acteur. Autrement dit, les effets de proximité peuvent varier selon les contextes et, si les individus étaient parfaitement mobiles, ces effets disparaîtraient. Chaque ville ou espace local combine de façon singulière des flux de trajectoires correspondant à des réseaux de structures différentes. Les flux et structures de réseaux les plus fréquents déterminent la force et la nature des liens locaux et donc les effets de proximité. Caractériser les effets de proximité produits par un site déterminé implique donc d'analyser les systèmes d'activités mais aussi la mobilité des habitants. 
Cela permet de comprendre ce qui différencie les types de systèmes industriels locaux que j'ai évoqués. Dans les districts italiens ou les sites français de même nature, la famille joue un rôle déterminant («le district comme réalité de production et le district comme ensemble de familles, comme unité de vie politique et sociale, etc., sont (...) intimement imbriqués », Capecchi, 1987, p.17) parce que les populations concernées sont plutôt sédentaires. Dans les systèmes industriels de haute technologie, les instituts de formation, les entreprises et les autres organisations professionnelles ou non professionnelles sont les cadres principaux de formation et de maintien des relations sociales parce que les populations concernées sont nettement plus mobiles et rassemblent des personnes ayant pour une bonne part passé leur enfance ailleurs. Encore existe-t-il entre les sites des différences importantes liées aux formes de concentration des populations et à leur mobilité. Ainsi dans des pôles d'enseignement supérieur français comme Toulouse et Grenoble, ou dans la Silicon Valley des débuts, les instituts de formation ont plus d'importance que dans des sites plus structurés autour de l'activité des entreprises comme la Silicon Valley actuelle, voire, dans une certaine mesure, à Sophia-Antipolis (si tant est que l'on montre que les entreprises qui y sont rassemblées forment des systèmes). Lorsqu'elles forment système, comme pour le textile dans le quartier du Sentier à Paris ou le montage et la distribution d'ordinateurs personnels dans la communauté chinoise de Los Angeles (Zhou, 1996), les concentrations d'activités dans certains secteurs de grandes agglomérations sont souvent structurées par des réseaux familiaux ou communautaires. Là encore, ce n'est pas la proximité physique en soi qui compte, ce sont les relations sociales qui rendent possible des engagements et des prises de risques.

On ne peut donc pas comprendre les effets de proximité en restant au seul niveau des organisations. Il faut pouvoir «descendre » au niveau des individus et de leurs réseaux et pouvoir remonter ensuite au niveau des organisations et, au-delà, au niveau des systèmes plus vastes. Les notions d'encastrement et de découplage permettent une telle navigation entre les niveaux d'analyse (Grossetti et Bès, 2003).

$\mathrm{Si}$ la proximité spatiale favorise, dans certaines conditions, l'apparition de coopérations entre les organisations, c'est donc principalement parce qu'elle favorise 
l'existence et la pérennisation de relations sociales sur la base desquelles ces coopérations peuvent s'établir.

\section{Une typologie des systèmes productifs locaux}

Le retour sur les «districts » et les «technopoles » permet de dégager des points communs et des différences.

Le point commun principal est que dans tous ces sites, la proximité spatiale (à l'échelle des agglomérations ou des bassins d'emploi) se traduit par l'existence de réseaux sociaux qui favorisent les échanges entre les organisations (entreprises, laboratoires), en particulier en ce qui concerne l'information technique, mais parfois aussi les marchés.

Les différences sont nombreuses.

D'un côté des petites entreprises de secteurs traditionnels, de l'autre des sociétés de «haute technologie» (i.e. qui ont une activité de R\&D) et des universités ou des organisations scientifiques. D'un côté des innovations «spontanées » (la mise au point du délainage à Mazamet, du moulage des plastiques à Oyonnax) largement partagées dès leur apparition, de l'autre une recherche organisée d'innovation, dont les auteurs cherchent à s'assurer au moins une exclusivité partielle, mais qui circule quand même pour partie par le jeu des réseaux. D’un côté des ouvriers, des techniciens et des petits patrons, de l'autre des cadres et des ingénieurs. D'un côté des sites en zone semi-rurale, de l'autre de grandes agglomérations. D'un côté des relations sociales structurés autour des familles, de l'autre des relations issues majoritairement de l'enseignement supérieur ou du travail. D'un côté une mise en commun des connaissances techniques et parfois des débouchés ou des fournisseurs, de l'autre une interface assurée en partie par des centres de recherche.

Résumons ces différentes caractéristiques : 
« Districts » et « technopoles »

\begin{tabular}{|c|c|c|}
\hline & « Districts» & « Technopoles» \\
\hline $\begin{array}{l}\text { Types d'organisations } \\
\text { productives }\end{array}$ & $\overline{\mathrm{PME}}$ & $\begin{array}{c}\text { Groupes, PME, organisations } \\
\text { scientifiques (universités, écoles, } \\
\text { laboratoires) }\end{array}$ \\
\hline R\&D & non & oui \\
\hline Innovation & 《spontanée » & organisée \\
\hline Localisation & zones semi-rurales, petites villes & $\begin{array}{c}\text { dans les grandes agglomérations } \\
\text { ou à proximité }\end{array}$ \\
\hline $\begin{array}{l}\text { Ressources échangées hors } \\
\text { marché localement }\end{array}$ & $\begin{array}{l}\text { information technique, } \\
\text { information sur les marchés }\end{array}$ & $\begin{array}{l}\text { information technique, sur les } \\
\text { partenaires }\end{array}$ \\
\hline $\begin{array}{l}\text { Ressources échangées localement } \\
\text { de façon marchande ou } \\
\text { contractuelle }\end{array}$ & prêts d'argent & $\begin{array}{c}\text { information technique, conseils, } \\
\text { prêts d'argent }\end{array}$ \\
\hline Coordination & $\begin{array}{c}\text { sur les salaires et les embauches } \\
\text { (limitation des surenchères } \\
\text { salariales) }\end{array}$ & $\begin{array}{c}\text { sur les salaires et les embauches } \\
\text { (limitations des surenchères } \\
\text { salariales) }\end{array}$ \\
\hline Expression politique & collectivités locales & collectivités locales \\
\hline Mobilité des populations & sédentaires & mobiles \\
\hline Relations sociales dominantes & familiales, d'école, de travail & d'université, de travail \\
\hline
\end{tabular}

Explicitons un peu les rubriques.

J'ai distingué les ressources échangées hors marché et celles qui font l'objet d'une transaction financière ou d'un accord formel. L'idée est que la proximité peut jouer sur les deux mais les modes de mesure sont différents. Il va de soi que le jeu des encastrements/découplages fonctionne aussi différemment. L'existence de transactions financières tend à favoriser des logiques de découplage, c'est-à-dire la reprise en main par le niveau des organisations de projets de coopérations élaborés au départ sur la base de relations entre individus.

La coordination désigne les relations qui dépassent le simple échange ou la collaboration sur une activité productive pour atteindre une forme de régulation collective, pouvant dans certains cas se concrétiser sous la forme d'un organisme intermédiaire (comité interprofessionnel par exemple). 
L'expression politique désigne l'accès de certains membres des organisations du système productif local à des responsabilités politiques, ce qui se traduit en général par la défense des intérêts de certaines des catégories impliquées dans le système productif (entrepreneurs, salariés, etc.). Tous les systèmes locaux ne débouchent pas nécessairement sur une expression politique, mais lorsque cela se produit, ce n'est évidemment pas sans effet sur le système.

La mobilité des populations est ici évidemment présentée de façon sommaire. Les personnes impliquées dans le système local sont plus ou moins mobiles mais elles ne le sont jamais toutes intégralement.

Enfin les lieux de formation des relations sont ceux qui apparaissent le plus fréquemment et constituent ainsi une «signature » possible pour certains systèmes, mais les relations peuvent avoir bien d'autres sources.

Cette liste de ressemblances et de différences suggère la possibilité d'étendre à d'autres types de configurations ce qui est commun aux deux types de concentrations industrielles envisagées ici. Il y en a au moins quatre.

1. Les systèmes de sous-traitance. Dans certains sites, un groupe industriel structure un réseau de sous-traitants situés à proximité. C'est le cas de Ford à Bordeaux (Carrincazeaux et Lung, 1998), à l'Aérospatiale à Toulouse (Talbot, 2001), de Peugeot à Sochaux, Michelin à Clermont, etc. Ces systèmes peuvent être qualifiés de SPL si, « toutes choses non spatiales qu'on ait pensé à mesurer égales par ailleurs », il y a plus d'échanges locaux (marchands ou non) qu'il n'y en aurait à distance. On pense ici au savoir technique ou à la coordination sur les embauches.

2. Les activités rares à marché mondial. Hollywood (Scott, 2000), la City de Londres ou la finance à Manhattan, la mode à Paris et de nombreux autres systèmes du même type rassemblent dans de grandes métropoles (souvent dans certains quartiers de ces métropoles) des métiers artisanaux formant système pour produire des bien rares aux débouchés internationaux. Là encore, les réseaux sociaux jouent un rôle essentiel dans la circulation des informations et des connaissances. 
3. Les services métropolitains. Les travaux de Jean-Marc Zuliani montrent que les services tertiaires aux entreprises (avocats d'affaires, conseils en communication, en gestion, etc.), constitués de petites structures, tendent à fonctionner en réseau grâce aux relations personnelles entretenues par les membres de ces structures (Zuliani, 1998). Ces réseaux ne sont évidemment pas uniquement locaux (comme tous ceux dont j'ai fait mention jusqu'ici d'ailleurs), mais un maillage local fait que ces structures font système et qu'au sein de ce système et au long de ces réseaux circulent des ressources (informations sur les clients, passages de relais, etc.) et peuvent émerger des innovations de type organisationnel.

4. Les systèmes agroalimentaires. Très souvent derrière une appellation contrôlée ou un produit régional «typé », l'on trouve des producteurs qui se concertent, s'échangent des savoirs techniques (la diffusion de la technique du micro-bullage dans le Madirannais, par exemple), tout en étant concurrents sur la qualité et les prix de leurs produits.

Récapitulons les différents types de SPL que nous avons rencontrés au cours de cette réflexion : 
Tableau 2

Les 6 types de SPL

\begin{tabular}{|c|c|c|c|c|c|c|}
\hline $\begin{array}{l}\text { Caractéristiq } \\
\text { ues }\end{array}$ & $\begin{array}{l}\text { «Dis- } \\
\text { tricts } »\end{array}$ & $\begin{array}{l}\text { « Techno- } \\
\text { poles » }\end{array}$ & $\begin{array}{c}\text { «sous- } \\
\text { traitance » }\end{array}$ & $\begin{array}{l}\text { « activités } \\
\text { rares » }\end{array}$ & $\begin{array}{l}\text { «ervices } \\
\text { métropo- } \\
\text { litains » }\end{array}$ & $\begin{array}{l}\text { « Système } \\
\text { s agro- } \\
\text { alim- } \\
\text { entaires » }\end{array}$ \\
\hline $\begin{array}{l}\text { Types } \\
\text { dominants } \\
\text { d'organi- } \\
\text { sations } \\
\text { productives }\end{array}$ & PME & $\begin{array}{c}\text { Groupes, PME, } \\
\text { organisations } \\
\text { scientifiques } \\
\text { (universités, écoles, } \\
\text { laboratoires) }\end{array}$ & Groupe, PME & PME, artisans & $\begin{array}{c}\text { PME, } \\
\text { Travailleurs } \\
\text { indépendants, } \\
\text { Professions } \\
\text { libérales }\end{array}$ & PME \\
\hline $\mathbf{R \& D}$ & non & oui & plus ou moins & non & non & non \\
\hline Innovation & $\begin{array}{c}\text { «spontanée } \\
»\end{array}$ & organisée & organisée & $\begin{array}{l}\text { organisée mais } \\
\text { sans R\&D } \\
\text { (activités de } \\
\text { création } \\
\text { artistique) }\end{array}$ & 《spontanée » & 《spontanée » \\
\hline Localisation & $\begin{array}{l}\text { zones semi- } \\
\text { rurales, } \\
\text { petites villes }\end{array}$ & $\begin{array}{c}\text { dans les grandes } \\
\text { agglomérations ou à } \\
\text { proximité }\end{array}$ & $\begin{array}{c}\text { indifférente } \\
\text { (plutôt agglos) }\end{array}$ & métropoles & métropoles & rural \\
\hline $\begin{array}{l}\text { Ressources } \\
\text { échangées } \\
\text { hors marché } \\
\text { localement }\end{array}$ & $\begin{array}{l}\text { information } \\
\text { technique, } \\
\text { information } \\
\text { sur les } \\
\text { marchés }\end{array}$ & $\begin{array}{l}\text { information } \\
\text { technique, sur les } \\
\text { partenaires }\end{array}$ & $\begin{array}{c}\text { information } \\
\text { technique }\end{array}$ & $\begin{array}{c}\text { information } \\
\text { technique, sur } \\
\text { les partenaires }\end{array}$ & $\begin{array}{l}\text { information sur } \\
\text { les partenaires }\end{array}$ & $\begin{array}{l}\text { information } \\
\text { technique, } \\
\text { information } \\
\text { sur les } \\
\text { marchés }\end{array}$ \\
\hline $\begin{array}{l}\text { Ressources } \\
\text { échangées } \\
\text { localement de } \\
\text { façon } \\
\text { marchande ou } \\
\text { contractuelle }\end{array}$ & $\begin{array}{l}\text { prêts } \\
\text { d'argent }\end{array}$ & $\begin{array}{c}\text { information } \\
\text { technique, conseils, } \\
\text { prêts }\end{array}$ & prêts d'argent & $\begin{array}{l}\text { prêts, conseils, } \\
\text { marques }\end{array}$ & prêts d'argent & prêts d'argent \\
\hline Coordination & $\begin{array}{c}\text { sur les } \\
\text { salaires et les } \\
\text { embauches } \\
\text { (limitation } \\
\text { des } \\
\text { surenchères } \\
\text { salariales) }\end{array}$ & $\begin{array}{c}\text { sur les } \\
\text { salaires et les } \\
\text { embauches } \\
\text { (limitation des } \\
\text { surenchères } \\
\text { salariales) }\end{array}$ & $\begin{array}{c}\text { sur les salaires } \\
\text { et les } \\
\text { embauches } \\
\text { (refus de } \\
\text { surenchère } \\
\text { salariale) }\end{array}$ & $\begin{array}{l}\text { sur les salaires } \\
\text { et les } \\
\text { embauches } \\
\text { (limitation des } \\
\text { surenchères } \\
\text { salariales), sur } \\
\text { l'image globale } \\
\text { de la « place » }\end{array}$ & sur les prix & $\begin{array}{c}\text { sur les } \\
\text { salaires et les } \\
\text { embauches } \\
\text { (limitation } \\
\text { des } \\
\text { surenchères } \\
\text { salariales), } \\
\text { sur l'image } \\
\text { globale de la } \\
\text { "place» } \\
\text { (normes de } \\
\text { qualité) }\end{array}$ \\
\hline $\begin{array}{l}\text { Expression } \\
\text { politique }\end{array}$ & $\begin{array}{l}\text { collectivités } \\
\text { locales }\end{array}$ & collectivités locales & $\begin{array}{l}\text { collectivités } \\
\text { locales }\end{array}$ & non ou faible & non ou faible & $\begin{array}{c}\text { collectivités } \\
\text { locales }\end{array}$ \\
\hline $\begin{array}{l}\text { Mobilité des } \\
\text { populations }\end{array}$ & sédentaires & mobiles & mélange & très mobiles & mobiles & $\begin{array}{l}\text { à dominante } \\
\text { sédentaire }\end{array}$ \\
\hline $\begin{array}{l}\text { Relations } \\
\text { sociales } \\
\text { dominantes }\end{array}$ & $\begin{array}{l}\text { familiales, } \\
\text { d'école, de } \\
\text { travail }\end{array}$ & $\begin{array}{l}\text { d'université, de } \\
\text { travail }\end{array}$ & $\begin{array}{l}\text { familiales, } \\
\text { d'école, de } \\
\text { travail }\end{array}$ & $\begin{array}{c}\text { professionnelle } \\
\mathrm{s}\end{array}$ & $\begin{array}{l}\text { d'université, de } \\
\text { travail }\end{array}$ & $\begin{array}{l}\text { familiales, } \\
\text { d'école, de } \\
\text { travail }\end{array}$ \\
\hline
\end{tabular}

\section{Conclusions et questions}


Le cadre esquissé ici permet de dégager un certain nombre de questions de recherche concernant les systèmes productifs locaux.

La première question est évidemment celle du rapport entre les relations locales et celles qui ne le sont pas, ou le sont moins. Un système productif local ne saurait être conçu comme un isolat. Il n'est qu'un lieu de densification des relations dans un réseau international. Les relations locales et non locales sont-elles complémentaires, concurrentes, sans aucun lien entre elles ? À partir de quel niveau de relations non locales la liaison avec l'extérieur est-elle efficace (Zimmermann, 2002) ?

La seconde question est celle des critères à partir desquels on peut dire que l'on a affaire à un système local. Le critère général qui vient à l'esprit est que l'on doit s'intéresser au système local à partir du moment où on ne peut plus comprendre les logiques et le fonctionnement des individus et des organisations présents sans y faire référence. Mais peut-on aller plus loin ? Peut-on définir des seuils, pourquoi pas quantitatifs, dans la densité des relations locales à partir desquels on pourrait travailler ? Ces seuils devraient-ils concerner les relations marchandes, non marchandes, la circulation des personnes, la structure des réseaux sociaux ? Comment utiliser ces seuils pour conforter ou amender la typologie ?

Troisième question: Dans quelle mesure un tel système correspond-il aux perceptions qu'en ont ceux qui y vivent? Où se situe ce qu'un historien des districts textiles appelle la «conscience de place» (Cazals, 1983), comment se forme-t-elle, s'exprime-t-elle, se mesure-t-elle? On peut supposer que l'émergence de lieux collectifs (organismes interprofessionnels locaux) ou la prise de pouvoir dans des instances locales peut favoriser l'émergence d'un mythe local du système, un discours récurrent repris par les différents acteurs et faisant référence pour le plus grand nombre, à l'intérieur comme à l'extérieur. On aura compris que, pour moi, l'expression politique et la conscience collective de l'existence du système ne sont pas nécessaires à son existence comme forme analysée par les chercheurs. Mais il est clair que ces dimensions supplémentaires, lorsqu'elles existent, peuvent influer fortement sur l'évolution du système. 
Quatrième question : Quels sont les niveaux pertinents du «local »? Par exemple, entre le local et le national, le niveau régional est certainement à creuser pour comprendre les logiques de certaines activités qui finissent par investir toute une région, au-delà des systèmes locaux. Existe-t-il des systèmes régionaux ailleurs que dans l'imaginaire des gestionnaires de régions?

Enfin, la cinquième question concerne l'innovation. La plupart des travaux empiriques montrent que les systèmes productifs locaux favorisent l'apparition d'innovations (technologiques ou sociales) ou en tout cas leur diffusion rapide au sein du système. On manque toutefois d'études réellement comparatives qui permettrait d'aller au-delà de cette convergence des analyses. Il faudrait en particulier parvenir à mettre en rapport les processus d'innovation avec la structure des systèmes (les réseaux des organisations et ceux des individus), à la fois sur le plan interne et sur le plan externe (les liens vers l'extérieur du système). On peut penser que certaines structures sont plus favorables que d'autres à l'innovation et même que, dans certains cas, les structures lui sont plutôt défavorables (prédominance de liens forts, peu de relations externes). Quelle interaction y a-t-il entre les structures relationnelles des systèmes et les processus d'innovation?

Malgré tous les efforts déjà accomplis, tant sur le plan empirique que théorique, il reste donc beaucoup à faire pour comprendre les systèmes productifs locaux. Il faut pour cela se résoudre à éliminer quelques explications mono-causales trop simples (les cultures locales, les savoirs tacites, etc.) et se donner les moyens, théoriques et méthodologiques, de faire le lien entre des niveaux différents d'action et d'analyse.

\section{Références}

Aydalot, P., 1986 (Ed.). Milieux innovateurs en Europe, GREMI.

Beccatini, G., 1989. Les districts industriels en Italie. In : Mariani M. et al., La Flexibilité en Italie. MIRE-TEN, Paris, pp. 261-271.

Beccatini, G., 1992. Le district marshallien : une notion socio-économique. In : Benko, G. et Lipietz, A., Les Régions qui gagnent. PUF, Paris, pp. 35-55.

Bellet, M., Boureille, B., 1985. Conditions d'émergence du développement local à partir d'un pôle productique : le cas de la région stéphanoise. Revue d'économie régionale et urbaine 4, 741-754.

Benko, G., Lipietz, A., 1992. Les Régions qui gagnent. PUF, Paris.

Benko, G., 1991. Géographie des technopôles. Masson, Paris. 
Bernardy, M., Boisgontier, P., 1988. Grains de technopoles, Micro-entreprises grenobloises et nouveaux espaces productifs. Presses Universitaires de Grenoble, Grenoble.

Bernardy, M., Boisgontier, P., 1996. La technopole, une certaine idée de la ville. L'Harmattan, Paris.

Bernardy, M., Boisgontier, P., 1976. Ingénierie urbaine, tertiaire supérieure : Lyon et Grenoble. Rapport DGRST, Septembre, $110 \mathrm{p}$.

Cappechi, V., 1987. Formation professionnelle flexible en Émilie-Romagne. Formation et emploi 19, 1987, 3-18.

Carrincazeaux, C., Lung, Y., 1998. La proximité dans l'organisation des activités de conception des produits automobiles. In : Bellet, M., Kirat, T. et Largeron, C. (Eds.), Approches multiformes de la proximité. Hermès, Paris, pp. 241-265.

Castells, M., (Ed.), 1984. High technology, space and society. Sage Publications, Urban affairs annual reviews, Londres.

Carrincazeaux, C., 1999. L'organisation spatiale de la recherche industrielle. Proximité et coordination des activités de R\&D des firmes. Thèse de doctorat en Sciences économiques (nouveau régime), Université Montesquieu-Bordeaux IV.

Cazals, R., 1983. Les révolutions industrielles à Mazamet, 1750-1900. Éditions La Découverte, Paris.

Courlet, C., Pecqueur, B., 1991. Systèmes locaux d'entreprises et externalités : un essai de typologie. Revue d'Économie Régionale et Urbaine 3/4, 391-406.

Estades, J., Joly, P.-B., Mangematin, V., 1996. Dynamique des relations industrielles dans les laboratoires d'un grand organisme public de recherche : coordination, apprentissage, réputation et confiance. Sociologie du Travail 3, 391-408.

Feldman, Maryann P., 1994. The geography of innovation, Economics of Science, Technology and Innovation 2. Kluwer Academic Publishers, Dordrecht, Boston, Londres.

Fischer, Claude S., 1982. To Dwell Among Friends, University of Chicago Press, Chicago.

Ganne, B., 1983. Gens du cuir, gens du papier. Éditions du CNRS, Paris.

Gilly, J.-P. Grossetti, M., 1993. Organisations, individus et territoires. Le cas des systèmes locaux d'innovation. Revue d'Économie Régionale et Urbaine 3, 449-468.

Granovetter, M., 1985. Economic action and social structure : the problem of embeddedness. American Journal of Sociology 91, 481-510.

Grossetti, M., 1995. Science, industrie et territoire. Presses Universitaires du Mirail, Toulouse.

Grossetti, M., 2001a. Les effets de proximité spatiale dans les relations entre organisations : une question d'encastrements. Espaces \& Sociétés 101-102, 203-219.

Grossetti, M., 2001b, Genèse de deux systèmes urbains d'innovation en France : Grenoble et Toulouse. Réalités Industrielles, Annales des Mines, ESKA Paris, pp. 68-72.

Grossetti, M., 2003. Relations sociales, espace et mobilités. Rapport pour le Plan Urbanisme Contruction Architecture, programme "Mobilités et territoires urbains", 150 pages.

Grossetti, M., Bès, M.-P., 2003. La dynamique des cercles et des réseaux. Encastrements et découplages. Revue d'Économie Industrielle 103, 43-58.

Grossetti, M., Bès, M.-P., 2002. Proximité spatiale et relations science-industrie : savoirs tacites ou encastrement (Polanyi ou Polanyi). Revue d'Économie Régionale et Urbaine 5 (II), 777-788.

Katz, John S., 1994. Geographical proximity and scientific collaboration. Scientometrics 31 (1), 31-43.

Maillat, D., Crevoisier, O.,Vasserot, J.-Y., 1992. Innovation et district industriel : l'Arc jurassien suisse, In : Maillat, D., Perrin, J.-C. (Eds.), Entreprises innovatrices et développement territorial. GREMI, EDES, Neuchâtel, pp. 105-126.

Mansfield, E., Jeong-Yeon, L., 1996. The modern university : contributor to industrial innovation and recipient of industrial R\&D support. Research policy 25, 1047-1058.

Marshall, A., 1890. Principes d'économie politique (traduction française 1906).

Perrin, J.-C., 1986. Les PME de haute technologie à Valbonne Sophia-Antipolis. Revue d'Économie Régionale et Urbaine 5, 629-643.

Planque, B., 1985. Le développement des activités à haute technologie et ses répercussions spatiales, l'exemple de la Silicon Valley. Revue d'Économie Régionale et Urbaine 5, 911-941.

Raveyre, M.-F. Saglio, J., 1984. Les systèmes industriels localisés : éléments pour une analyse sociologique des ensembles de PME industriels. Sociologie du travail 2, 157-175.

Rogers, E., Larsen, J., 1984. Silicon Valley fever : growth of high technology culture. Basic books, New York. 
Saxenian, A., 1981. Silicon chips and spatial structure : the industrial basis of urbanisation in Santa Clara County, California. Institute of Urban and Regional Planning. WP 345, University of California, Berkeley.

Saxenian, A., 1990a. Regional networks and the resurgence of Silicon Valley. Californian Management. Review 33, 89-112.

Saxenian, A., 1990b. The origins and dynamics of production networks in Silicon Valley. Working paper 516. Institute of Urban and Regional Development, University of California, Berkeley, $35 \mathrm{p}$.

Saxenian, A., 1992. Contrasting patterns of business organization in Silicon Valley. Environment and Planning D 10, p. 377-391.

Saxenian, A., 1994. Regional Advantage. Harvard University Press, Cambridge (Mass.).

Scott, Allen, J., 2000. The Cultural Economy of Cities. Essays on the Geography of Image-Producing Industries. Sage, London.

Scott, Allen J., 1985. High technology industries and territorial development : the rise of the Orange County complex, 1955-1984. Department of geography, UCLA, Los Angeles.

Talbot, D., 2001. Mondialisation et dynamiques des coordinations inter-firmes : le cas dans la soustraitance aéronautique. Sciences de la Société 54, 153-165.

Wellman, B., 1979. The community question : the intimate networks of east yorkers. American Journal of Sociology 84 (5), 1201-1231.

Wellman, B., 1996. Are personal communities local ? A Dumptarian reconsideration. Social Networks $18,347-354$

White, Harrison, C., 1992. Identity and Control. A structural theory of action. Princeton University Press, Princeton.

Zhou, Y., 1996. Inter-firm linkages, Ethnic Networks, and Territorial Agglomeration : Chinese Computer Firms in Los Angeles. Papers in Regional Science 75 (3), 265-291.

Zimmermann, J.-B., 2002. «Grappes d'entreprises » et «petits mondes »: une affaire de proximités. Revue Économique 53 (3), 517-524.

Zuliani, J.-M., 1998. Effets de proximité et développement métropolitain des services de haut niveau : le cas de Toulouse. Sud-Ouest Européen 2, 33-46.

Zuliani, J.-M., Grossetti, M., 2004. Les liaisons science - industrie dans les aires métropolitaines : logiques sectorielles et encastrements territoriaux - Toulouse. In : Régis Guillaume (dir.), Systèmes productifs et dynamiques territoriales, regards croisés au Québec et dans le Sud-Ouest français. (À paraître.) 